\title{
Acolhimento à população de lésbicas, gays, bissexuais e transgêneros na atenção básica
}

\section{Welcoming the lesbians, gays, bisexual and transgenean population in basic care}

\author{
Ellen Thallita Hill Araújo' • Geovani Ferreira de Sousa² • José Arnaldo Moreira de Carvalho Júnior ${ }^{3}$ Francisco \\ Gaunié de Sousa Pessôa ${ }^{4}$ Luana Kelle Batista Moura ${ }^{5}$
}

\begin{abstract}
RESUMO
Objetivo: analisar os significados atribuídos por enfermeiros da atenção primária acerca do conhecimento e prática do acolhimento à população de Lésbicas, Gays, Bissexuais e Transgêneros (LGBT). Métodos: pesquisa exploratória e descritiva, de abordagem qualitativa, realizada com vinte enfermeiros que atuam na Estratégia Saúde da Família (ESF) de uma cidade do Nordeste do Brasil. Utilizou-se um roteiro semiestruturado para entrevistas realizadas em janeiro a março de 2019. Os depoimentos transcritos foram processados no software IRaMuTeQ e analisados pela Classificação Hierárquica Descendente. Resultados: a Classificação Hierárquica Descendente permitiu a identificação e a análise dos domínios textuais em um dendograma, que ilustrou as relações entre seis classes nomeadas conforme seu conteúdo a partir de três eixos. Os significados atribuídos por enfermeiros encontraram-se ancorados na necessidade de aprimoramento para qualidade no processo de acolhimento à população LGBT. Conclusão: É relevante investir em estudos que reforçem a ampliação das propostas de humanização e dos debates sobre o acolhimento e práticas de saúde a essa população.
\end{abstract}

Descritores: Acolhimento, Enfermagem, Minorias sexuais e de gênero,Atenção Básica.

\begin{abstract}
Objective: to analyze the meanings attributed by nurses in primary care about the knowledge and practice of welcoming the LGBT population. Methods: exploratory and descriptive research, with a qualitative approach, carried out with twenty nurses who work in the Family Health Strategy (FHS) in a city in the Northeast of Brazil. A semi-structured script was used for interviews conducted from January to March 2019. The transcribed testimonies were processed using the IRaMuTeQ software and analyzed using the Descending Hierarchical Classification. Results: the Descending Hierarchical Classification allowed the identification and analysis of textual domains in a dendogram, which illustrated the relationships between six classes named according to their content based on three axes. The meanings attributed by nurses were anchored in the need to improve quality in the welcoming process for the LGBT population. Conclusion: It is necessary to recognize that the LGBT population is not only formed by a multiplicity of orientations and gender identities and to invest in studies that reinforce the expansion of the humanization proposals for the reception of this population.
\end{abstract}

Keywords: Reception; Nursing; Sexual and gender minorities; Basic Attention.

I Enfermeira. Mestre em Saúde da Família, Centro Universitário UNINOVAFAPI,Teresina (PI), Brasil.E-mail: ellen.araújo@unimedteresina.com.br

2 Enfermeiro. Pós-graduação em urgência e emergência pelo Centro Universitário UNINOVAFAPI,Teresina (PI), Brasil. E-mail: geovaniferreira.sousa@live.com

3 Enfermeiro. Doutorando em Educação em Ciências e Saúde pelo Programa de Pós-graduação em Educação em Ciências e Saúde- NUTES/UFRJ. Rio de Janeiro/RJ, Brasil.E-mail: arnaldocarvalhojr@hotmail.com

4 Enfermeiro. Graduado em Enfermagem pela Faculdade do Piauí- FAPI.Teresina/PI, Brasil. E-mail: gaunie.sousa I0@hotmail.com

5 Cirurgiã-Dentista. Pós-Doutora em Saúde Coletiva pela Universidade Federal do Rio Grande do Norte (UFRN). Doutora em Endodontia pela Universidade de Ribeirão Preto (UNAERP). Docente Titular do Programa de Mestrado Profissional em Saúde da Família do Centro Universitário UNINOVAFAPI.Teresina-PI-Brasil. E-mail: luanamoura@uninovafapi.edu.br 


\section{INTRODUÇÃO}

A diversidade sexual e de gênero tem sido um tema constantemente discutido na mídia. Gênero é um conceito subjetivo, é definido como elemento constitutivo das relações sociais baseadas nas diferenças percebidas entre os sexos. Desse modo, relaciona-se à maneira como as sociedades lidam com a percepção dos corpos humanos ${ }^{1,2}$.

Em 1990, iniciou-se o movimento para defender as diferenças de gêneros, definido pela sigla LGBT, contemplando em sua composição os segmentos de Lésbicas, Gays, Bissexuais e Transgêneros ${ }^{3}$.

No grupo dos LGBT as pessoas transgênero, incluídas travestis e transexuais, estão entre as mais sujeitas a sofrer preconceitos, discriminações e violências. Possuem identidades de gênero diversas àquelas impostas pelos padrões heteronormativos vigentes na sociedade ${ }^{4}$.

O acolhimento dessa população deve ser um compromisso de resposta às necessidades dos cidadãos que procuram os serviços de saúde, com a construção de uma relação de confiança, vinculo e compromisso, respeitando a equidade e procurando entender as necessidades de saúde ${ }^{5}$.

Em 20I I, no Brasil, foi formulada a Política Nacional de Saúde Integral de Lésbicas, Gays, Bissexuais, Travestis e Transexuais (PNSILGBT).A PNSILGBT é uma iniciativa para a construção de mais equidade no Sistema Único de Saúde (SUS), sendo um dos seus objetivos, a garantia do acesso ao Processo Transexualizador, que representa um complexo de expedientes assistenciais direcionados à atenção e ao cuidado de transexuais e travestis que tenham o desejo de realizar mudanças corporais através da adequação da aparência física e da função de suas características sexuais ${ }^{6}$.

Desta forma, o acolhimento dessa comunidade na atenção básica de saúde começou a ser assumido como compromisso profissional e ético, para garantia da universalidade, acessibilidade e redução das desigualdades relacionadas à saúde ${ }^{7}$.

Diante dessa política, um dos papeis do enfermeiro é orientar corretamente o usuário LGBT quanto aos seus direitos e deveres dentro do sistema único de saúde ${ }^{8}$.

No entanto, percebe-se na literatura, sobretudo Latino-americana, lacunas no que concerne ao acolhimento à população LGBT pelos profissionais de enfermagem atuantes na Atenção Primária. Baseado nisto, a pergunta que norteou o estudo foi: "Qual os significados atribuídos por enfermeiros da atenção primária sobre o acolhimento à população LGBT?". Desta forma, objetivou-se analisar o conhecimento e prática do acolhimento e assistência dos enfermeiros da atenção primária à população LGBT.

\section{MÉTODOS}

Tratou-se de um estudo exploratório e descritivo, de abordagem qualitativa, fundamentado na Teoria Representacional do Significado (TRS) de Ogden e Richards. De acordo com os pressupostos da TRS os significados têm inúmeros conceitos e adotou-se aquele que apresenta o sentido representacional. Essa teoria vem sendo utilizada como recurso metodológico em estudo internacional na interpretação de termos científicos e nacionais da área de saúde sobre significados, com ênfase na enfermagem 9,10 .

A coleta de dados foi conduzida por um roteiro semiestruturado aplicado através de uma entrevista com 20 enfermeiros que atuam na Estratégia Saúde da Família (ESF) de uma cidade do Nordeste do Brasil. Foram incluídos profissionais que atuavam há mais de um ano cadastrados na Unidade Básica de Saúde (UBS). Foram excluídos do estudo enfermeiros que se encontravam de licença do trabalho ou de férias durante o período de coleta de dados.

Para seleção dos participantes foram sorteadas, pelo aplicativo Random, vinte Unidades Básicas de Saúde das 38 existentes, as quais foram visitadas para entrevista do enfermeiro participante do estudo.

Todos os enfermeiros foram entrevistados em suas unidades de trabalho. Realizou-se previamente o convite e agendamento da data e horário conforme disponibilidade do participante. Procedeu-se à coleta dos dados entre os meses de janeiro e março de 2019. As entrevistas foram realizadas em espaço privativo, em encontro único e gravadas em áudio utilizando aplicativo próprio em celular com 25 minutos de tempo médio de entrevista. Ao término os participantes ouvirem e validarão seus relatos, não houveram desistências ou alterações.

Utilizou-se o software IRaMuTeQ (acrônimo de Interface de R pour les Analyses Multidimensionnelles de Textes et de Questionnaires) para o processamento dos dados. Optou-se por utilizar um software nessa etapa, baseado na transparência e sistemática conferida ao processo".

Os dados foram tratados utilizando-se o método da classificação hierárquica descendente segundo o qual os textos são classificados em função de seus respectivos vocabulários, e o conjunto deles se divide pela frequência das formas reduzidas. A partir de matrizes que cruzaram segmentos de textos e palavras (repetidos testes qui-quadrado), aplicou-se o método da classificação hierárquica descendente, e foi obtida uma classificação estável e definitiva. Essa classificação permitiu a obtenção de classes de segmentos de texto com vocabulário semelhante entre si, mas, ao mesmo tempo, diferente dos segmentos de texto das outras classes ${ }^{12}$.

O estudo atendeu criteriosamente aos preceitos éticos nacionais em pesquisa envolvendo seres humanos da 
Resolução 466/2012 do Conselho Nacional de Saúde, sendo aprovado pelo Comitê de Ética em Pesquisa da Instituição Proponente.

Todos os participantes foram informados quanto aos objetivos do estudo e registraram a concordância por meio da assinatura do Termo de Consentimento Livre e Esclarecido, em duas vias. $\mathrm{O}$ anonimato dos participantes e a confidencialidade dos conteúdos foram preservados por meio da codificação dos depoimentos coletados, tendo sido utilizada a letra "E", a qual representou "Enfermeiros", seguida por um número arábico sequencial a realização de cada entrevista:“(EI), (E2) ...(E20).

\section{RESULTADOS}

Participaram da pesquisa 20 enfermeiros das ESF, sendo todos do sexo feminino, com média de idade de 33 anos, tempo médio de atuação profissional de 5 anos e tempo de formação com variação entre 5 e $I 5$ anos. Em relação a realização de pós-graduações, todos possuíam especializações (lato sensu). No que diz respeito às capacitações sobre acolhimento a população LBGT, nenhum enfermeiro declarou ter realizado.

Com relação aos depoimentos, o IRaMuTeQ reconheceu a separação do corpus em 214 Unidades de Contexto Elementares (UCEs) a partir de 282 segmentos de texto. Foram apontadas 1176 ocorrências, com um apro- veitamento de $75 \%$ do total do corpus textual. A CHD admitiu a assimilação e análise dos domínios textuais, além da interpretação dos significados atribuídos pelos enfermeiros, nomeando-as em seus respectivos sentidos em seis classes originadas de três eixos.

O primeiro eixo formado originou a classe 2 , a qual é relacionada ao conhecimento do enfermeiro acerca do atendimento da população LGBT na Atenção Básica. O segundo eixo formou a classe 6 , com conteúdo retratando os motivos para procura da Unidade Básica de Saúde pela população LGBT.A terceira partição (eixo) possui duas ramificações (sub-divisões), representadas pelas classes I e 5, que abordam as dificuldades para o atendimento da população LGBT nas Unidades Básicas de Saúde (UBS).As classes 3 e 4 emergiram de uma segunda partição do corpus, originando o eixo com conteúdo retratando o acolhimento da população LGBT na atenção básica e as atendimento qualificado à essa população nas UBS, respectivamente (Figura I).

\section{As classes e seus significados}

A Classe I, intitula-se "Dificuldades para $\circ$ atendimento da população LGBT nas Unidades Básicas de Saúde (UBS)". Seu conteúdo é focado no papel do Enfermeiro, e sua preocupação em socializar dessa população nas atividades das UBS. Nesta classe, os enfermeiros relatam a necessidade de um aprimoramento para qualidade

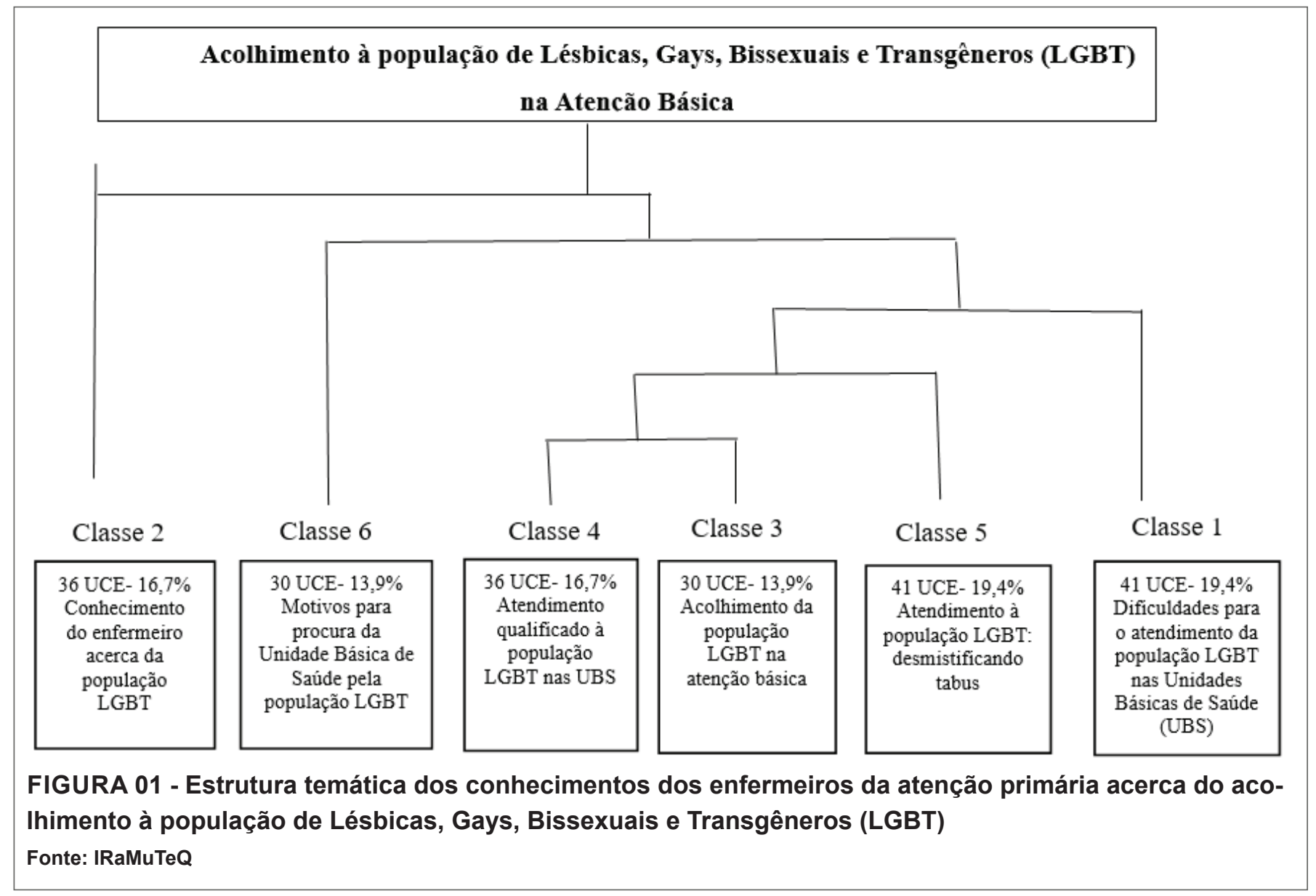


no processo de acolhimento no Sistema único de Saúde (SUS), com enfoque na mudança nas formas de pensar e agir, reconhecimento de cada indivíduo como único, singularidade no cuidado, respeitando e reconhecendo a sexualidade do outro.

[...] e algumas coisas não se encaixam no serviço para essa população, seria necessário realmente um atendimento específico[...] (E2)

[..] Não acho necessário fazer um aprimoramento, dessas coisas, eu já acho que é como se fosse um preconceito[...](E4)

[.] precisamos recorrer que precisamos de mais formação, aperfeiçoamento e informação para segurança no atendimento desse público[...] (EI6)

[...] e enfermeiro na sua formação básica tem muito pouco conhecimento a respeito desse tema[...](E/8)

$\mathrm{Na}$ Classe 2, intitulada "Conhecimento do enfermeiro acerca da população LGBT", os participantes atribuem conceitos à população LGBT baseado na sua vivência e experiência enquanto profissional. Além disso, reconhecem a importância do atendimento a essa população para a melhoria da saúde e qualidade de vida. Todavia, ainda se percebe uma extensa discussão e as controvérsias em torno do significado da sigla LGBT.

É inegável a dúvida que surge entre os profissionais quando $O$ assunto é identidade de gênero. A compreensão da sexualidade, que é diversa, e suas relações sociais da atualidade requer uma discussão mais abrangente nas abordagens da saúde, como pode-se observar na predominância dos vocábulos ou símbolos: sexo, masculino, gostar, entender, saber, não sei, mesmo. Tais conceitos são controversos, tendo em vista que o objetivo final é fortalecer uma rede de cuidados a essa população na atenção primária em saúde.

[...] em resumo o que significa, pra mim são pessoas que gostam do mesmo sexo[...](EI)

[...]lesbicas que são as mulheres, gays e transexuais eu não sei[...](E9)

[...]Lésbicas, gays, e não sei o resto.Aqui no posto eles são iguais todos os pacientes que buscam atendimento[...](E/3)

[...]LGBT eu acho que é gay, bissexual e transexual, esse $L$ eu não sei o que é não $[. .].(E / 7)$

Da Classe 3, emergiu o "Acolhimento da população LGBT na atenção básica”. Diante das políticas que visam garantir a todos os cidadãos cuidados humanizado, livre de qualquer preconceito e discriminação com base na orientação sexual e identidade de gênero, o enfermeiro tem o papel de acolher e orientar corretamente o usuário LGBT quanto aos seus direitos e deveres dentro do sistema único de saúde. Embora corretas, tais orientações ainda são pontuais e desarticuladas o que demonstra fragilidades na formação e conhecimento dos participantes.

[...] No acolhimento eu chego e pergunto pra eles, porquê as vezes a gente pensa que é um jeito e na hora é outro, as vezes eles não se aceitam [...] $(E 3)$

[...]com muita conversa eu entendo que com muita conversa pode ser que eles possam confiar [...](E5)

[...] faço a identificação do paciente independentemente do tipo de identidade sexual que ele tiver[...] (E8)

[...] tratar de forma normal como se fosse uma pessoa qualquer[...] (EIO)

$\mathrm{Na}$ Classe 4, denominada "Atendimento qualificado à população LGBT nas UBS" os participantes revelaram suas fragilidades quando reconhecem a especificidade necessária para cuidar da população LGBT. As dificuldades relacionadas às questões sociais e falta de investimentos em educação continuada comprometem as competências e habilidades na assistência a esse grupo no âmbito da atenção primária.

[...]Tenho uma necessidade de aprimorar para atender essa população[...] (EI)

[...]Não tem nenhum programa voltado pra esse público e não tive treinamento sobre isso[...](E2)

[...] por que vai ter aprimoramento para este tipo? Porque é pra tratar como outro, aqui nós tratamos de planejamento familiar[...](E8)

[..] algumas coisas não se encaixam no serviço para essa população, seria necessário um treinamento[...] (El8)

A Classe 5 revelou "Atendimento à população LGBT: desmistificando tabus". Historicamente, é compreendido que a população LGBT sofre preconceito e perseguição por sua orientação sexual e identidade de gênero, e mais recentemente, sobretudo, em decorrência do estigma da AIDS, afastando-os, assim, de ambientes de cuidados com a saúde.

A maioria das mulheres lésbicas ou bissexuais não revelam sua orientação sexual nas consultas e entre as que revelam consideram mais rápido o atendimento na UBS. A dificuldade dessas mulheres se assumirem homo ou bissexuais e a negação do risco de contrair as doenças sexualmente transmissíveis estão entre os principais motivos que levam à menor procura pelos serviços de saúde da atenção primária.

Com relação aos homossexuais masculinos, como são as principais vítimas da violência e homicídios homofóbicos leva a estigma do medo de atendimento discriminatório nas unidades, constrangimentos, condutas inadequadas, conotações preconceituosas ou mesmo ofensas verbais proferidas pelos profissionais.

[...] como eles não se protegem, os mais fixos procuraram o postinho de saúde, através de alguma doença sexualmente transmissiveis[...] (EI)

[...] a gente já teve algumas divergências, algumas complicações, o público feminino LGBT não entende que tem que fazer a citologia preventiva[..] (E2)

$[\ldots .$.$] eles se encobrem eles não gostam de demonstrar o$ 
que realmente eles são, eles querem atendimento, mas tem receio de descriminação[...](E4)

[...] Não tem nenhum programa voltado pra esse público com relação a realização do preventivo[...](EI 7)

[..]são pessoas resistentes, eles não procuram muitos os serviços de saúde [...](E20)

Por fim, na Classe 6, foi denominada "Motivos para procura da Unidade Básica de Saúde pela população LGBT", os participantes verbalizaram quais as principais queixas da população LGBT quando buscam atendimento nas UBS.A análise qualitativa dos vocábulos mais evocados a buscar por hormônios, prevenção e o risco de contrair as infecções sexualmente transmissíveis estão entre os principais motivos que levam à procura dessa população pelos serviços da atenção primária.

Apesar do reconhecimento dos profissionais sobre o ganho para esse segmento populacional da regulamentação do processo transsexualizador no SUS, chama atenção de que ele não soluciona o problema do acesso precário na atenção básica e da qualidade da atenção em todos os níveis do sistema.

[...] as vezes eles procuram hormônios, eles querem os anticoncepcionais, eles querem usar os injetáveis aí é meio complicado a liberação[...](E2)

[...] eu já tive alguns que procuraram pra liberação de anticoncepcionais injetáveis, assim a gente já teve algumas divergências [...](E6)

[...] eles buscam quando estão sentindo algum problema a nivel de DST [...](E7)

[...]Principal, doenças sexualmente transmissiveis, sempre aconselho o teste rápido[...](E/4)

[...]Geralmente aqui e pra fazer teste rápido[...](E/5)

Assim, por meio da classificação hierárquica descendente (distribuição das classes e seus conteúdos), percebe-se que o conhecimento e atuação do enfermeiro voltada ao atendimento da população LGBT é deficiente para atuação holística, embora permita instrumentalizar a comunidade em questões pontuais acerca das infecções sexualmente transmissíveis e planejamento familiar, observando uma dificuldade na ruptura dos tabus.

\section{DISCUSSÃO}

Alguns participantes demonstraram desconhecimento ao expressar seu entendimento acerca da sigla "LGBT", apresentando dúvidas durante as entrevistas, quando questionados acerca do tema. Houve falas pausadas e momentos de silêncio, demostrando, uma possível fragilidade conceitual. Manifestaram-se ainda restrição de ideias com relação à temática, observando-se que o assunto pode remeter algo negativo e constrangedor aos profissionais.

Estudos ratificaram que os profissionais não sabem como proceder, mesmo com o cenário atual de diversifi- cação de gênero, pois não tiveram durante a sua formação acadêmica ou formação profissional, a oportunidade de discutir a temática da diversidade sexual e suas ligações com a saúde da família ${ }^{13}$.

Uma revisão de estudos empíricos evidenciou que preconceitos interferem na compreensão e imparcialidade dos atendimentos da população LGBT na atenção básica, aspectos destacados sugerem que um número significativo de profissionais de saúde sente-se desconfortáveis na prestação de cuidados aos clientes gays e lésbicas ${ }^{14}$.

Em trabalhos qualitativos a população LGBT relatou ter vivenciado tratamentos discriminatórios de saúde, incluindo procedimentos desnecessários, referiram atendimento recusados pelos profissionais de saúde quando os mesmos tomaram conhecimento da história de gênero ${ }^{15}$.

Uma pesquisa realizada na Califórnia sobre a taxas de comportamentos discriminatórios envolvendo a população LGBT nos serviços de saúde, revelou que 10\% dos participantes thes foram negados encaminhamentos, $65 \%$ ouviram comentários depreciativos e $34 \%$ testemunharam tratamento discriminatório ${ }^{16}$.

Todavia, é fundamental que as ações da atenção básica voltadas a este público não sejam transitórias e pontuais, mas que englobem a realidade que os mesmos estão inseridos. A necessidade de sensibilização de profissionais da saúde para o atendimento não discriminatório da população LGBT ainda é um dos temas mais recorrentes nas políticas públicas de saúde formuladas para esses segmentos ${ }^{17}$.

Contrapondo-se ao exposto, uma pesquisa realizada com estudantes de enfermagem acerca da sexualidade, apresentou que os acadêmicos possuem segurança no relato da sua percepção sobre diversidade de gênero e interesse em ampliar seus conhecimentos devido à relevância do tema para a futura atuação profissional ${ }^{18}$.

Outras pesquisas revelaram que após a população LGBT manifestar a sua orientação sexual, os profissionais deram encaminhamento ao atendimento mais rápido ou não solicitaram exames, o que pode comprometer o atendimento. Além disso, sugeriram que as lésbicas são menos propensas a obter cuidados de rotina, o que inclui exames ginecológicos, mesmo tendo o risco aumentado de câncer de mama e do endométrio devido à alta incidência de nuliparidade ${ }^{19}$.

Esta população está exposta a doenças crônicas em percentuais significativos e refere que as taxas de notificação para doenças crônicas são maiores para populações homossexuais. Para mulheres heterossexuais, as taxas chegam a $70 \%$ e, para lésbicas, $80 \%$, em que se destacam as doenças cardiovasculares, câncer de pulmão e câncer de mama, todas associadas ao tabagismo ${ }^{14}$.

A literatura traz evidências apontando que a popula- 
ção LGBT tem menos acesso aos serviços e cuidados em saúde, cerca de $40 \%$ de pacientes LGBT nos Estados Unidos citam a falta de acesso a saúde como uma barreira ao cuidado e uma grande quantidade relata a recusa de tratamento, cuidado precário e abuso verbal por parte dos profissionais de saúde e como resultado muitos pacientes evitam tratamento médico, mesmo nas situações de emergência ${ }^{20}$.

\section{CONSIDERAÇÕES FINAIS}

Os significados atribuídos pelos enfermeiros da atenção primária acerca do conhecimento e prática do acoIhimento à população LGBT estiveram relacionados aos símbolos (sexo, masculino, mulher, gostar, transmissível, doença, população, saúde, acolhimento, aceitar, dificuldade, normal, público e procurar), ou seja, vocábulos significantes evocados dos depoimentos dos participantes e utilizados para compor o corpus originando as Unidades de Contexto Elementar.

De uma forma geral, de acordo com a Teoria Repre- sentacional do Significado os significados no pensamento apontam que é preciso garantir a igualdade no acesso a saúde em uma perspectiva integral, livre de preconceitos, com qualidade e onde a orientação sexual e a identidade de gênero não sejam determinantes negativos.

As limitações desse estudo são relacionadas ao método adotado, o qual limita a generalização dos resultados para além do contexto subjetivo dos participantes. A ampliação do cenários e dos participantes possibilitará oferecer mais subsídios para que os significados apreendidos sejam aprofundados.

Dadas as peculiaridades que envolvem $\circ$ assunto em questão, é preciso reconhecer que a população LGBT é formada por uma multiplicidade de orientações. Contudo, torna-se relevante investir em estudos que reforçem a ampliação das propostas de humanização e dos debates sobre o acolhimento e práticas de saúde a essa população.

Conflito de interesses: nenhum declarado. 


\section{REFERÊNCIAS}

I. Lemes LB. Reconhecer as diferenças para superar os (pre)conceitos de gênero e de sexualidade na educação. Rev. Estud. Fem.2018; 26(3):I-5. doi:I0.1590/I806-9584$2018 \mathrm{v} 26 \mathrm{n} 353016$.

2. Gomes R, Murta D, Facchini R, Meneghel SN. Gende and sexual rights: their implications on health and healthcare. Ciência \& Saúde Coletiva. 2018; 23(6):1997-2005. doi:I0.1590/|4|3-8|2320|8236.048720|8.

3. Brasil. Ministério da Saúde (MS). Transexualidade e Travestilidade na Saúde. Brasília: MS; 2015.

4. Hirschle TMR, Maciel SC, Amorim GK. Representaciones sociales sobre el cuerpo y satisfacción sexual de mujeres mastectomizadas y sus parejas. Temas psicol. 2018;26(I): 457-468. doi: 10.9788/TP20 18. I- I8Pt

5. Popadiuk GS, Oliveira DC, Signorelli MC. The National Policy for Comprehensive Health of Lesbians, Gays, Bisexuals and Transgender (LGBT) and access to the Sex Reassignment Process in the Brazilian Unified Health System (SUS): progress and challenges. Ciência \& Saúde Coletiva. 2017; 22(5): I509-I520.

6. Hughes RL, Damin C, Heiden-Rootes K.Where's the LGBT in integrated care research? A systematic review. Fam Syst Health. 2017;35(3):308-319. doi: 10.1037/fsh0000290.

7. Rivers I, Gonzalez C, Nodin N, Peel E, Tyler A. LGBT people and suicidality in youth: $A$ qualitative study of perceptions of risk and protective circumstances. Soc Sci Med. 2018; 212:I-8. doi: 10.1016/j.socscimed.2018.06.040.

8. Radix A, Maingi S. LGBT Cultural Competence and Interventions to Help Oncology Nurses and Other Health Care Providers. Semin Oncol Nurs. 2018;34(I):80-89. doi: 10.1016/j.soncn.2017.12.005.

9. Ogden CK, Richards IA. O significado de significado: um estudo da influência da linguagem sobre o pensamento e sobre a ciência do simbolismo. Rio de Janeiro: Zahar, 1976.

10. Remsen D. The use and limits of scientific names in biological informatics. ZooKeys.2016; I I3(550):207-23.

I I.Almeida CAPL, Oliveira LMM, Franca MJO, Martins AP, Oli- veira ADS, Rocha SCV, et al. Risk factors and meaning of violence against the elderly from the perspective of nursing students. SYLWAN. 2017; I6I (7): I50-162.

12. Kami MTM, Larocca LM, Chaves MMN, Lowen IMV, Souza VMP, Goto DYN. Working in the street clinic: use of IRAMUTEQ software on the support of qualitative research. Esc.Anna Nery. 2016;20(3): e20160069.

13. Santos AR, Santos RMM, Souza ML, Boery RNSO, Sena ELS, Yarid SD. Implicações bioéticas no atendimento de saúde ao público LGBTT. Rev bioét. 20I5;23(2):4I9- 26.

14. Sousa AFL, Queiroz AAFLN, Oliveira LB, Moura MEB, Batista OMA, Andrade D. Social representations of biosecurity in nursing: occupational health and preventive care. Rev Bras Enferm. 2016;69(5): 864-87I.

15. Costa LD, Barros AD, Prado EAJ, Sousa MF, Cavadinha ET, Mendonça AVM. Competência Cultural e Atenção à Saúde da população de lésbicas, gays, bissexuais travestis e transexuais (LGBT) Tempus, actas de saúde colet, 20 I 7; I I (I): I05-II9.

16. Portz JD, Retrum JH,Wright LA, Boggs JM,Wilkins S, Grimm $C$ et al. Assessing capacity for providing culturally competent services to LGBT older adults. J Gerontol Soc Work 20I4; 57: 305-32I.

17. Leal CBM, Porto AO, Barbosa CB, Fernandes SS, Fernandes ESF, Viana TBP. Nursing Assistance to the Adolescent Public in Primary Care. Revista Enfermagem Atual In Derme. 2018; 86: I-9.

18. Santos LV, Campos MPA, Ribeiro AO, Mattos MCT. Sexualidade humana: conhecimentos dos acadêmicos de enfermagem. Esc Anna Nery Rev Enferm. 20 I 7; I (2):303-6.

19. Facchini R, Barbosa R. Dossiê Saúde das Mulheres Lésbicas: promoção da equidade e da integralidade. Belo Horizonte: Rede Feminista de Saúde; 2016.

20. Cavalcanti AC.Acolhimento nos Serviços de Saúde à População LGBT: Uma Revisão Integrativa. Revista do Congresso Brasileiro de Ciências da Saúde. 20I6; I (I):I - 9.

Recebido: $2020-02-24$ Aceito: 2020-04- 15 